\title{
All NSR-Models in Terms of Bosonic Strings: An Explicit Derivation
}

\author{
Georg Keller \\ Theoretical Physics, ETH-Hönggerberg, CH-8093 Zürich, Switzerland
}

\begin{abstract}
We construct the open and closed string NSR-models in terms of $D \geqq 15$ bosonic string theories. All anticommuting NSR-operators are obtained after fermionizing 4 bosonic dimensions, and the NSR-Hilbert spaces are embedded as linear subspaces of the bosonic Hilbert spaces. We thus show the existence of various $10 D$ supersymmetric sectors of the state spaces of $D=26$ consistent bosonic strings.
\end{abstract}

\section{Introduction}

String theories containing space-time-bosonic and -fermionic degrees of freedom [1] are known to be interpretable as $2 D$ (two dimensional) quantum field theories. Those theories leading to space-time-fermions are based, in the old formalism of Green and Schwarz [1], on theories possessing a priori $2 D$-fermions only. Because $2 D$ bosonization and fermionization $[2,3]$ have become a tool of investigation in $2 D$ models, one might wonder if it is possible to construct the NSR (NeveuSchwarz-Ramond)-models [1] in terms of the consistent $D=26$ bosonic string theory. This has been anticipated by Freund [4] and partially achieved by the authors of $[5]^{1}$.

In this paper, motivated by [5], we use $D \geqq 15$ closed compactified and open bosonic strings, which are not necessarily consistent, in order to construct explicitly all the operators characterizing the open and closed NSR-models in terms of purely bosonic ones. We make use of the fermionization method of [3] and of results on the quantum equivalence of various fermionic realizations of Virasoro algebras in two dimensions [6]. We describe the possible choices of subspaces of the bosonic Hilbert space which become the Hilbert spaces of the spinning string. That is, we construct $10 D$ supersymmetric sectors of the closed or open $D=26$ bosonic string.

However, it turns out that it does not seem to be more efficient to calculate, for instance, superstring scattering amplitudes using results obtained from bosonic

\footnotetext{
${ }^{1}$ However, the NSR models were not treated by [5]
} 
string theory. The same conclusion applies to heterotic string theories [7] and to all string theories based on the new formalism of Green and Schwarz [1], because the transitions from the old to the new formalism, and from the "candidate" superstring in $[5]^{2}$ to the new formalism are quite impractical. These "candidate" superstrings are string theories which are formulated on the Ramond sector only. We will describe and subsequently classify them. Their classification is done by means of an analysis of the Lorentz transformation properties of their massless groundstates. The crucial technical ingredients will be the triality properties of $S O(8)$, since $S O(8)$ constitutes the relevant classification subgroup of the Lorentz group for massless states in all $10 \mathrm{D}$ theories.

This paper is organized as follows: In the next section, we will review all the necessary formulae of closed compactified and open bosonic strings and also those of the NSR-models. Section 3 is devoted to the construction of anticommuting operators from commuting ones, following [3]. We will write down all the relevant commutation relations which are needed in Sect. 4 to understand the emergence of the various NSR-models. Finally, in the last part of this paper, we will investigate the candidate superstrings of ref. [5]. In particular, we will show how chirality is assigned to a fixed set of groundstates.

\section{Bosonic and Fermionic Formulae}

One of the most satisfactory ways of deriving the constraints and equations of motion both for the open and closed spinning and (compactified) bosonic strings starts with the action [8] in $2 D$ Minkowski space

$$
\begin{aligned}
S= & \frac{1}{4 \pi \alpha^{\prime}} \int_{M} d^{2} \xi e \cdot\left\{\frac{1}{2} g^{\alpha \beta} \partial_{\alpha} X^{\mu} \partial_{\beta} X_{\mu}+\frac{i}{2} \bar{\psi}^{\mu} \varrho^{a} \partial_{\alpha} \psi_{\mu} e_{a}^{\alpha}\right. \\
& \left.-\frac{1}{2}\left(\bar{\chi}_{\alpha} \varrho^{b} \varrho^{a} \psi^{\mu}\right)\left(\partial_{\beta} X_{\mu}-\frac{1}{4} \bar{\chi}_{\beta} \psi_{\mu}\right) e_{a}^{\alpha} e_{b}{ }^{\beta}\right\}
\end{aligned}
$$

where, for $\mu=0, \ldots, D-1$,

$$
\begin{aligned}
& X^{\mu}=2 D \text { real scalar field }, \\
& \psi^{\mu}=2 D \text { Majorana-Grassmann spinor field },
\end{aligned}
$$

and $e_{\alpha}{ }^{a}$ is the zweibein field, and $\chi_{\alpha}$ is its $2 D$ supersymmetry partner (for each $\alpha, \chi_{\alpha}$ is a Majorana-Grassmann spinor field).

The $2 D \gamma$-matrices in a Majorana representation are

$$
\varrho^{0}=\left(\begin{array}{c}
{ }^{-i} \\
i
\end{array}\right), \quad \varrho^{1}=\left(\begin{array}{l}
i \\
i
\end{array}\right), \quad \varrho^{5}=i \varrho^{0} \varrho^{1}=\left(\begin{array}{cc}
i & \\
& -i
\end{array}\right) .
$$

2 These string theories, which are likely to be superstring theories, were found in [5], although some of the arguments used seem invalid. But after completion of this work we received [11] which is the revised and more detailed version of [5] 
The charge-conjugation operator is given by $C=1$, and

$$
\eta_{a b} e_{\alpha}^{a} e_{\beta}^{b}=\mathrm{g}_{\alpha \beta}, \quad e=\operatorname{det}\left(e_{\alpha}^{a}\right), \quad \eta_{a b}=\left(\begin{array}{cc}
1 & \\
& -1
\end{array}\right) \text {. }
$$

From now on we set $\alpha^{\prime}=\frac{1}{2}$. We vary $S$ in (2.1) with respect to $X^{\mu}, \psi^{\mu}, \chi_{\alpha}, e_{\alpha}{ }^{a}$ and use the symmetries of $(2.1)$ to choose the superconformal gauge

$$
\begin{aligned}
\chi_{\alpha} & =i \varrho^{a} \tilde{\eta} e_{\alpha a}, \\
e_{\alpha}{ }^{a} & =e^{\phi} \delta_{\alpha}^{a}
\end{aligned}
$$

where $\tilde{\eta}$ is an arbitrary $2 D$ Majorana-Grassmann spinor field. In the superconformal gauge, the equations of motion become

$$
\begin{aligned}
\square X^{\mu} & =0, \\
\left(\partial_{0}-i \varrho^{5} \partial_{1}\right) \tilde{\psi}^{\mu} & =0,
\end{aligned}
$$

and the constraints read

$$
\begin{aligned}
\tilde{\psi}^{\mu}\left(\partial_{0}+i \varrho^{5} \partial_{1}\right) X_{\mu} & =0, \\
\left(\left(\partial_{0} \pm \partial_{1}\right) X\right)^{2}+i \widetilde{\psi}^{\mu}\left(\partial_{0} \pm \partial_{1}\right) \widetilde{\psi}_{\mu} & =0,
\end{aligned}
$$

where $\partial_{0}=\partial / \partial \xi^{0}, \partial_{1}=\partial / \partial \xi^{1}, \square=\partial_{0}^{2}-\partial_{1}^{2}, \tilde{\psi}^{\mu}=e^{\phi / 2} \psi^{\mu}$. Exploiting the residual conformal invariance and $2 D$ global supersymmetry of $(2.1)-(2.1 \mathrm{c})$ we are free to choose finally the light-cone-gauge

$$
X^{+}=q^{+}+p^{+} \tau, \quad \tilde{\psi}^{+}=0 .
$$

We impose the suitable boundary conditions on $X^{\mu}$ and $\widetilde{\psi}^{\mu}$ and obtain ${ }^{3}$ : bosonic string field $X^{\mu}$

physical space-time:

$$
\begin{aligned}
& X^{+}=q^{+}+p^{+} \tau, \\
& X^{-}=q^{-}+p^{-} \tau+i \sum_{n \neq 0} \frac{\alpha_{n}^{-}}{n} z^{-n}+i \sum_{n \neq 0} \frac{\bar{\alpha}_{n}^{-}}{n} \bar{z}^{-n}, \\
& X^{i}=q^{i}+p^{i} \tau+i \sum_{n \neq 0} \frac{\alpha_{n}^{i}}{n} z^{-n}+i \sum_{n \neq 0} \frac{\bar{\alpha}_{n}^{i}}{n} \bar{z}^{-n}, \quad i=2, \ldots, d-1(d \leqq D),
\end{aligned}
$$

with $\alpha_{0}^{i} \equiv \bar{\alpha}_{0}^{i}:=\frac{1}{2} p^{i}, z=e^{i(\tau-\sigma)}, \bar{z}=e^{i(\tau+\sigma)}$.

For open strings : $\alpha_{n}^{i,-} \equiv \bar{\alpha}_{n}^{i,-}$, and $d \leqq D$.

For closed strings: $\alpha_{n}^{i,-}$ and $\bar{\alpha}_{n}^{i,-}$ are independent, and $d \leqq D$.

${ }^{3}$ The conventions chosen are: $X^{ \pm}=\frac{1}{\sqrt{2}}\left(X^{0} \pm X^{1}\right) ; d^{2} \xi=d \sigma d \tau$; the open strings are parametrized by $\mathbb{R} \times[0, \pi]$, the closed ones by $\mathbb{R} \times[0,2 \pi]$, where $\mathbb{R} \times[a, b]$ denote the $\tau$ - and the $\sigma$-intervals respectively; $Y^{\mu} X_{\mu}=Y^{+} X^{-}+Y^{-} X^{+}-Y^{i} X^{i}$ 
compactified dimensions (for closed strings only): $I=d, \ldots, D-1$,

$$
\begin{aligned}
& X^{I}=X_{L}^{I}+X_{R}^{I}=q^{I}+p^{I} \tau+2 L^{I} \sigma+i \sum_{n \neq 0} \frac{\alpha_{n}^{I}}{n} z^{-n}+i \sum_{n \neq 0} \frac{\bar{\alpha}_{n}^{I}}{n} \bar{z}^{-n}, \\
& X_{L}^{I}=q_{L}^{I}+p_{L}^{I}(\tau+\sigma)+i \sum_{n \neq 0} \frac{\bar{\alpha}_{n}^{I}}{n} \bar{z}^{-n}, \\
& X_{R}^{I}=q_{R}^{I}+p_{R}^{I}(\tau-\sigma)+i \sum_{n \neq 0} \frac{\alpha_{n}^{I}}{n} z^{-n},
\end{aligned}
$$

with $\alpha_{0}^{I}:=p_{R}^{I}, \bar{\alpha}_{0}^{I}:=p_{L}^{I}$, and

$$
2 L^{I}=p_{L}^{I}-p_{R}^{I}, \quad p^{I}=p_{L}^{I}+p_{R}^{I},
$$

and $\alpha_{n}^{I}, \bar{\alpha}_{n}^{I}$ independent, for $n \in Z$.

Thus uncompactified closed strings can be thought of as having $p_{L}^{I}=p_{R}^{I}$.

The open string can be compactified by changing $\partial_{\sigma} X^{I}(\sigma=0, \tau)=\partial_{\sigma} X^{I}(\sigma=\pi, \tau)$ $=0$ into $\partial_{\sigma} X^{I}(\sigma=0, \tau)=\partial_{\sigma} X^{I}(\sigma=\pi, \tau)=\Gamma$, where $\Gamma$ lies in some lattice $A$. But it is sufficient for our purposes to take $\Gamma \approx 0$ and just consider some

remaining dimensions (for open strings): $I=d, \ldots, D-1$ :

$$
\begin{gathered}
X^{I}=q^{I}+p^{I} \tau+i \sum_{n \neq 0} \frac{\alpha_{n}^{I}}{n} z^{-n}+i \sum_{n \neq 0} \frac{\bar{\alpha}_{n}^{I}}{n} \bar{z}^{-n}, \\
\text { with } \alpha_{n}^{I} \equiv \bar{\alpha}_{n}^{I}, \quad \text { and } \quad \alpha_{0}^{I} \equiv \bar{\alpha}_{0}^{I}=\frac{1}{2} p^{I} .
\end{gathered}
$$

fermionic string field

$$
\begin{aligned}
& \frac{1}{\sqrt{2}} \tilde{\psi}^{+}=0, \\
& \frac{1}{\sqrt{2}} \tilde{\psi}^{-}=\sum_{v \in I} d_{v}^{-} z^{-v}+\sum_{v \in I} \bar{d}_{v}^{-} \bar{z}^{-v}, \\
& \frac{1}{\sqrt{2}} \tilde{\psi}^{i, J}=\sum_{v \in I} d_{v}^{i, J} z^{-v}+\sum_{v \in I} \bar{d}_{v}^{i, J} \bar{z}^{-v},\left\{\begin{array}{l}
J=d, \ldots, D-1 \\
i=2, \ldots, d-1
\end{array} .\right.
\end{aligned}
$$

Here, the two sets of indices $\{i\}$ and $\{J\}$ are introduced only for convenience; physically there is no a priori difference between $\widetilde{\psi}^{i}$ and $\tilde{\psi}^{J}$. The index set $I$ is

$$
\begin{aligned}
& I=Z, \text { for R (Ramond) boundary conditions } \\
& I=Z+\frac{1}{2}, \text { for NS (Neveu-Schwarz) boundary conditions . }
\end{aligned}
$$

For open strings: $d_{v}^{-, i, I} \equiv \bar{d}_{v}^{-, i, I}$.

For closed strings: $d_{v}^{-, i, I}$ and $\bar{d}_{v}^{-, i, I}$ are independent.

In the usual way one finds for the dependent variables $\alpha_{n}^{-}, \bar{\alpha}_{n}^{-}, d_{v}^{-}, \bar{d}_{v}^{-}$, and $p^{-}$the 
following expressions ${ }^{4}$ :

$$
\begin{aligned}
& \alpha_{n}^{-}=\frac{1}{p^{+}}\left\{\sum_{m \in Z} \alpha_{n+m}^{i, I} \alpha_{-m}^{i, I}+\sum_{v} d_{n+v}^{i, I} d_{-v}^{i, I}(-v)\right\}, \\
& \bar{\alpha}_{n}^{-}=\frac{1}{p^{+}}\left\{\sum_{m \in Z} \bar{\alpha}_{n+m}^{i, I} \bar{\alpha}_{-m}^{i, I}+\sum_{v} \bar{d}_{n+v}^{i, I} \bar{d}_{-v}^{i, I}(-v)\right\}, \\
& d_{v}^{-}=\frac{2}{p^{+}}\left\{\sum_{m \in Z} d_{v+m}^{i, I} \alpha_{-m}^{i, I}\right\}, \\
& \bar{d}_{v}^{-}=\frac{2}{p^{+}}\left\{\sum_{m \in Z} \bar{d}_{v+m}^{i, I} \bar{\alpha}_{-m}^{i, I}\right\},
\end{aligned}
$$

and finally (for open strings: replace $p_{R}^{I}$ and $p_{L}^{I}$ by $\frac{1}{2} p^{I}$ )

$$
\begin{aligned}
& \frac{1}{8}\left(2 p^{+} p^{-}-\left(p^{i}\right)^{2}\right)=\frac{1}{8} m^{2}=N_{\alpha}+N_{d}+\frac{1}{2} p_{R}^{2}-c, \\
& \frac{1}{8}\left(2 p^{+} p^{-}-\left(p^{i}\right)^{2}\right)=\frac{1}{8} \bar{m}^{2}=\bar{N}_{\alpha}+\bar{N}_{d}+\frac{1}{2} p_{L}^{2}-\bar{c},
\end{aligned}
$$

where for right movers (unbarred oscillators)

$$
\begin{aligned}
& N_{\alpha}=\sum_{n>0} \alpha_{-n}^{i} \alpha_{n}^{i}+\sum_{n>0} \alpha_{-n}^{I} \alpha_{n}^{I} \equiv N_{\alpha}^{i}+N_{\alpha}^{I}, \\
& N_{d}=\sum_{\nu \geqq 0} d_{-v}^{i, I} d_{v}^{i, I} \cdot v .
\end{aligned}
$$

The analogous formulae hold for the left movers (barred oscillators). Thus we have for closed strings:
(a) $m^{2}=\bar{m}^{2} \Rightarrow N_{\alpha}+N_{d}+\frac{1}{2} p_{R}^{2}-c=\bar{N}_{\alpha}+\bar{N}_{d}+\frac{1}{2} p_{L}^{2}-\bar{c}$
(b) if uncompactified: $p_{R}=p_{L}=0$, and thus $i=2, \ldots, D-1$.

for open strings:

(a) here $m^{2}=\bar{m}^{2}$ gives no constraints because $c=\bar{c}, N_{\alpha}=\bar{N}_{\alpha}, N_{d}=\bar{N}_{d}, p_{L}=p_{R}$,

(b) if $d=D$, i.e. $i=2, \ldots, D-1: \frac{1}{8} m^{2}=N_{\alpha}+N_{d}-c$,

(c) if $d<D: \frac{1}{8} m^{2}=N_{\alpha}+N_{d}+\frac{1}{2} \tilde{p}^{2}-c$, where $\tilde{p}^{I} \equiv \frac{1}{2} p^{I}$.

The numbers $c, \bar{c}$ are normal ordering constants which are introduced after quantization $[1,7]$ :

$$
\begin{aligned}
{\left[\alpha_{n}^{i, I}, \alpha_{m}^{j, J}\right] } & \left.=n \delta_{n+m, 0}\left(\delta^{i j}+\delta^{I J}\right)\right\} \begin{array}{l}
\text { both for left } \\
\text { and right movers }
\end{array} \\
\left\{d_{v}^{i, I}, d_{\mu}^{j, J}\right\} & =\delta_{v+\mu, 0}\left(\delta^{i j}+\delta^{I J}\right) \quad \\
{\left[\alpha_{n}^{k}, \bar{\alpha}_{m}^{p}\right] } & =\left\{d_{v}^{i}, \bar{d}_{\mu}^{j}\right\}=0=\left[q_{L}^{I}, p_{R}^{J}\right], \\
{\left[q^{-}, p^{+}\right] } & =-i, \\
{\left[q^{i}, p^{j}\right] } & =i \delta^{i j}, \\
{\left[q_{L}^{I}, p_{L}^{J}\right] } & =\frac{1}{2} i \delta^{I J}=\left[q_{R}^{I}, p_{R}^{J}\right], \\
\left(\alpha_{n}^{I, i}\right)^{+} & =\alpha_{-n}^{I, i}, \quad\left(d_{v}^{i, I}\right)^{+}=d_{-v}^{i, I}, \quad \text { also for left movers. }
\end{aligned}
$$

\footnotetext{
${ }^{4}$ For ease of notation summation over repeated indices will always be understood
} 
Requiring closure of the Poincare group $P_{+}^{\uparrow}$ in the physical dimensions yields that for purely bosonic strings $\left(d_{v}=\bar{d}_{v}=0\right)$ :

for spinning strings:

$$
D=26 \text { and } c=\bar{c}=1 \text {. }
$$

$$
D=10 \text { and }
$$

$\left.\begin{array}{lllll}c & \text { or } \quad \bar{c}=0, & \text { for } & \text { R-b.c. } \\ c & \text { or } & \bar{c}=\frac{1}{2}, & \text { for } & \text { NS-b.c. }\end{array}\right\}$ imposed on $\sum_{v} d_{v} z^{-v}$ or $\sum_{v} \bar{d}_{v} \bar{z}^{-v}$,

thus getting (right-b.c., left-b.c.) $=(\mathrm{NS}, \mathrm{NS})-,(\mathrm{NS}, \mathrm{R})-,(\mathrm{R}, \mathrm{NS})-,(\mathrm{R}, \mathrm{R})$-models for closed and (R)-, (NS)-models for open strings.

Our method to construct the NSR-models form bosonic strings works for all bosonic theories with $c \geqq \frac{1}{2}, \bar{c} \geqq \frac{1}{2}$. But we will do the actual computations only in the special case $c=\bar{c}=1$. We will never require the closure of the bosonic Lorentz algebra, but we need at least $D=15$ dimensions. Thus the special case of consistent bosonic strings is automatically included in our treatment.

\section{Fermionization}

In order to get eight $2 D$ fermionic fields analogous to $\tilde{\psi}^{i}(z), i=1, \ldots, 8$, [Eq. (2.6)] from compactified bosonic strings, we take, according to [3], $D-d \geqq 5$ compactified dimensions 4 of which will be used in the following. Let us denote them by $I=1, \ldots, 4$.

Since everything we will do can be carried out separately for the right and left moving sectors of closed strings, and since identical results hold for open bosonic strings with $D-d \geqq 5$, we will restrict our attention to the right movers of the closed string.

Let us define, for right moving closed strings,

$$
\begin{aligned}
Q^{I}(z): & =2 q^{I}-i p^{I} \log z+i \sum_{n<0} \frac{\alpha_{n}^{I}}{n} z^{-n}+i \sum_{n>0} \frac{\alpha_{n}^{I}}{n} z^{-n} \\
& =2 q^{I}-i p^{I} \log z+Q_{<}^{I}+Q_{>}^{I},
\end{aligned}
$$

where $q^{I}=q_{R}^{I}, p^{I}=p_{R}^{I}$, and $z=e^{i(\tau-\sigma)}, \sigma \in[0,2 \pi]$.

For open strings, we write

$$
Q^{I}(z):=2 q^{I}-i \tilde{p}^{I} \log z+i \sum_{n<0} \frac{\alpha_{n}^{I}}{n} z^{-n}+i \sum_{n>0} \frac{\alpha_{n}^{I}}{n} z^{-n},
$$

where $\tilde{p}^{J} \equiv \frac{1}{2} p^{J}$ [cf. (2.12)] and thus $\left[q^{I}, \tilde{p}^{J}\right]=\frac{1}{2} i \delta^{I J}$, and $z=e^{i \tau}$.

Thus, all the commutation relations of the operators occurring in the expansion of $Q^{I}(z)$ in Eq. (3.1) are identical to those of the mode-operators of $Q^{I}(z)$ in Eq. (3.2).

Then, taking the standard orthonormal basis of $\mathbb{R}^{4},\left\{e_{i} \mid e_{i} \cdot e_{j}=\delta_{i j}\right\}$, we use the notation 


$$
\begin{aligned}
& \left\{ \pm e_{i}\right\} \equiv\left\{\lambda_{V}\right\}=\{\text { vector weights of } S O(8)\} \\
& \left\{\frac{1}{2} \sum_{i=1}^{4}\left( \pm e_{i}\right) \mid \#-=\text { odd }\right\} \equiv\left\{\lambda_{s}\right\}=\text { one set of spinor weights of } S O(8) \\
& \left\{\frac{1}{2} \sum_{i=1}^{4}\left( \pm e_{i}\right) \mid \#-=\text { even }\right\} \equiv\left\{\lambda_{\bar{s}}\right\}=\text { the other set of spinor weights of } S O(8) \\
& \left\{ \pm e_{i} \pm e_{j} \mid i \neq j\right\}=\text { generators of the root lattice } \Lambda_{R} \text { of } S O(8)
\end{aligned}
$$

Now we define the operator-valued fields

$$
B(e, z)=z^{e^{2} / 2}: e^{i e \cdot Q(z)}: \equiv z^{e^{2} / 2} e^{i e \cdot Q<} e^{2 i e q} z^{e \cdot p} e^{i e \cdot Q>},
$$

where we restrict our attention to $e \in\left\{\lambda_{V}\right\}$. Formally we expand $B(e, z)$ as a Laurent series (where we continue analytically $\tau \in \mathbb{R} \mapsto \tau \in \mathbb{C}$ )

and the index set is

$$
B(e, z):=\sum_{v \in I} z^{-v} B_{v}^{e}
$$

$$
\left.\begin{array}{lll}
I=Z, & \text { for } & \text { R-b.c. } \\
I=Z+\frac{1}{2}, & \text { for } & \text { NS-b.c. }
\end{array}\right\} \text { imposed on } B(e, z) .
$$

In order to have uniquely defined mode operators $B_{v}^{e}$, we need to know the allowed values of p. From (3.3), (3.4) we see that the domains of definition $D(B)$ are:

$$
\begin{aligned}
D(B)_{R} & =\left\{|\mathbf{p}, \boldsymbol{\alpha}\rangle \mid e \cdot p+\frac{1}{2} \in Z\right\}, \quad \text { for } \quad \text { R-b.c. } \\
& =\left\{|\mathbf{p}, \boldsymbol{\alpha}\rangle \mid \mathbf{p} \in\left\{\lambda_{s}+\Lambda_{R}\right\} \cup\left\{\lambda_{\vec{s}}+\Lambda_{R}\right\}\right\} ; \\
D(B)_{N S} & =\left\{|\mathbf{p}, \boldsymbol{\alpha}\rangle \mid e \cdot p+\frac{1}{2} \in Z+\frac{1}{2}\right\}, \quad \text { for NS-b.c. } \\
& =\left\{|\mathbf{p}, \boldsymbol{\alpha}\rangle \mid \mathbf{p} \in\left\{\lambda_{V}+\Lambda_{R}\right\} \cup \Lambda_{R}\right\},
\end{aligned}
$$

where $|\alpha\rangle$ denotes any oscillator-state. This is consistent, because, as we will see below, $B(e, z)$ and $B_{v}^{e}$ translate $|\mathbf{p}, \boldsymbol{\alpha}\rangle$ into $\sum_{\boldsymbol{\beta}} c(\boldsymbol{\alpha}, \boldsymbol{\beta} ; \mathbf{p}, \mathbf{e}) \cdot|\mathbf{p}+\mathbf{e}, \boldsymbol{\beta}\rangle$, and from the
above we note that

$$
|\mathbf{p}, \boldsymbol{\alpha}\rangle \in D(B) \Leftrightarrow B(e, z)|\mathbf{p}, \boldsymbol{\alpha}\rangle \in D(B),
$$

both for R- and NS-b.c. Furthermore $D(B)_{\mathrm{NS}} \perp D(B)_{\mathrm{R}}$.

Using $\left(\alpha_{n}^{I}\right)^{+}=\alpha_{-n}^{I}$, we get that $\left(B_{v}^{e}\right)^{+}=B_{-v}^{-e}$.

We note that the explicit form of $B_{v}^{e}$, which we will not bother to write down, because it is not needed here, crucially depends on which $|\mathbf{p}\rangle B(e, z)$ is acting.

Let us define the Virasoro generators,

$$
\begin{aligned}
& L_{n}:=\frac{1}{2} \sum_{m \in Z}: \alpha_{n+m}^{I} \alpha_{-m}^{I}:, \quad n \neq 0, \\
& L_{0}:=\frac{1}{2} \sum_{m \in Z}: \alpha_{+m}^{I} \alpha_{-m}^{I}:=\frac{1}{2}\left(p^{2}\right)+\sum_{m=1}^{\infty} \alpha_{-m}^{I} \alpha_{m}^{I}, \quad n=0,
\end{aligned}
$$


where $\alpha_{0}^{I}=p^{I}$, cf. (2.4), and in case of open strings $p^{I} \mapsto \tilde{p}^{I}$ because $\alpha_{0}^{I}=\tilde{p}^{I}$, cf. (2.5). If we take $L_{0}$ to be the Hamiltonian, we see that:

$R$-sector :

$$
L_{0} \geqq \frac{1}{2} ; \quad L_{0}|v\rangle=\frac{1}{2}|v\rangle \Leftrightarrow|v\rangle \in\left\{\left|\mathbf{p}=\lambda_{s}, \boldsymbol{\alpha}=0\right\rangle\right\} \cup\left\{\left|\mathbf{p}=\lambda_{\bar{s}}, \boldsymbol{\alpha}=0\right\rangle\right\} .
$$

Thus the vacuum is 16 -fold degenerate.

NS-sector :

$$
L_{0} \geqq 0 ; \quad L_{0}|v\rangle=0 \Leftrightarrow|v\rangle=|\mathbf{p}=0, \boldsymbol{\alpha}=0\rangle .
$$

Thus $|0\rangle$ is the unique vacuum.

With (3.3), (3.4), and (3.6) one obtains ${ }^{6}$ :

$$
\begin{aligned}
{\left[f \cdot p, B_{v}^{e}\right] } & =(e \cdot f) B_{v}^{e}, \quad \mathbf{f} \in \mathbb{R}^{4}, \\
{\left[N, B_{v}^{e}\right] } & =B_{v}^{e}\left(-\frac{1}{2}-v-e \cdot p\right), \\
{\left[L_{0}, B_{v}^{e}\right] } & =-v B_{v}^{e}, \\
\Rightarrow B_{v}^{e}|v\rangle & =0, \quad v>0 .
\end{aligned}
$$

Taking $\mathbf{e}, \mathbf{f} \in\left\{\lambda_{V}\right\}$, using contour-integration methods, one gets (see ref. [3])

$$
\begin{aligned}
{\left[B_{v}^{e}, B_{\mu}^{f}\right] } & =0, \quad e \cdot f=0, \\
\left\{B_{v}^{e}, B_{\mu}^{-e}\right\} & =\delta_{v+\mu, 0}, \\
\left\{B_{v}^{e}, B_{\mu}^{e}\right\} & =0 .
\end{aligned}
$$

Introducing now ad hoc the second part of the anticommuting algebraic structure [3], namely the operator cocycle,

$$
\begin{aligned}
\left\{c_{e} \mid c_{e} c_{f}\right. & =(-1)^{e \cdot f+1} c_{f} c_{e}, \quad \mathbf{e}, \mathbf{f} \in\left\{\lambda_{V}\right\} ; \\
\left(c_{e}\right)^{+} & =c_{-e} ; \\
c_{e} c_{-e} & =1\},
\end{aligned}
$$

which can be represented in terms of Dirac matrices, we get the desired result

$$
\left\{c_{e} B_{v}^{e}, c_{f} B_{\mu}^{f}\right\}=\delta_{v+\mu, 0} \delta^{e+f, 0} .
$$

Using operator product expansion we obtain

$$
\begin{gathered}
\circ B(e, w) B(-e, w) \circ:=\lim _{z \rightarrow w}\{B(e, z) B(-e, w)-\langle v|B(e, z) B(-e, w)| v\rangle\} \\
=e \cdot P(w) \equiv e \cdot i w \frac{d}{d w} Q(w) \equiv e \cdot \sum_{n \in Z} \alpha_{n} z^{-n}, \\
\circ B(e, w) B(f, w) \circ=B(e+f, w), \quad e \cdot f=0, \\
\circ B(e, w) B(e, w) \circ=0,
\end{gathered}
$$

where $|v\rangle$ stands for either $|0\rangle$ or one of the vacua of the $R$-sector.

${ }^{6}$ A sample calculation is presented in Appendix A 
Finally we want to define selfadjoint fermionic field operators. Let, for $j=1, \ldots, 4$,

$$
\begin{aligned}
H^{j}(z) & :=\frac{1}{\sqrt{2}}\left(c_{e_{j}} B\left(e_{j}, z\right)+c_{-e_{j}} B\left(-e_{j}, z\right)\right), \\
H^{4+j}(z) & :=\frac{-i}{\sqrt{2}}\left(c_{e_{j}} B\left(e_{j}, z\right)-c_{-e_{j}} B\left(-e_{j}, z\right)\right) .
\end{aligned}
$$

Since $B(e, z)^{+}=B\left(-e, \frac{1}{z}^{*}\right)$ we see that exactly in $2 D$ Minkowski space, where $|z|$ $=1$ and thus $\frac{1}{z}^{*}=z$, we have

$$
\left.\begin{array}{rl}
H^{j}(z)^{+} & =H^{j}(z) \\
H^{j}(z): & =\sum_{v} H_{v}^{j} z^{-v}, \quad \text { on } \quad D(H) \equiv D(B)
\end{array}\right\} j=1, \ldots, 8,
$$

\section{The NSR-Models}

In this section we will make use of the fermionization/bosonization formula (3.13) in order to derive Eqs. (4.8) which constitute the essential step from compactified bosonic dimensions towards the anticommuting part of the NSR models.

The first equation of (4.8), i.e. the Ramond-type expression, was also derived in [5]. In contrast to our methods those authors plugged Eq. (3.13) into the bosonic form of $L_{m}$ [i.e. into the left-hand side of (4.8)] and subsequently employed $\xi$-function regularization to determine the extra contribution $\frac{1}{2}$ for $L_{0}^{\text {ferm. }}$. Instead, we take recourse to the representation theories of $S O(8)$ and of Virasoro algebra, using the results of [6] on the quantum equivalence of seemingly different $2 D$ quantum field theories. This approach seems to be a rather natural one, since one of our aims is to prove the quantum equivalence of $L_{m}^{\text {bos. }}$ [left-hand side of Eq. (4.8)] with $L_{m}^{\text {ferm. }}$ [right-hand side of Eq. (4.8)] on a Hilbert space to be determined.

Using Eqs. (3.13), (3.16) we see that

$$
P^{j}(z) \equiv e_{j} \cdot P(z)=\frac{i}{2} \circ H^{k}(z)\left(T^{j+4, j}\right)_{k l} H^{l}(z) \circ, \quad j=1, \ldots, 4,
$$


where

$$
\left(T^{i j}\right)_{k l}=\left(\delta^{i, k} \delta^{j, l}-\delta^{j, k} \delta^{i, l}\right), \quad i, j=1, \ldots, 8,
$$

are the generators of the $S O(8)$ vector-representation (e.g. $i>j)$. The $P^{j}(z)$ with their moments

$$
p_{n}^{j}:=\frac{1}{2 \pi i} \oint \frac{d z}{z} P^{j}(z) z^{+n}
$$

form a generalized Cartan-subalgebra of the associated affine Lie algebra $S \hat{O}(8)$, with $p_{n}^{i} \equiv \alpha_{n}^{I}$. The full Kac-Moody algebra $S \hat{O}(8)$ is generated by the currents

$$
T^{i j}(z):=\frac{i}{2} \circ H^{k}(z)\left(T^{i j}\right)_{k l} H^{l}(z) \circ
$$

or, more precisely, by their moments

$$
T_{n}^{i j}:=\frac{1}{2 \pi i} \oint \frac{d z}{z} T^{i j}(z) z^{+n},
$$

which satisfy the $S \hat{O}(8)$ commutation relations

$$
\left[T_{n}^{i j}, T_{m}^{p q}\right]=i\left(T_{n+m}^{i q} \delta^{j, p}-T_{n+m}^{i p} \delta^{j, q}+T_{n+m}^{j p} \delta^{i, q}-T_{n+m}^{j q} \delta^{i, p}\right)+k \cdot n \cdot \delta^{i, p} \delta^{j, q} \delta_{n+m, 0} .
$$

Here $k$ is the standard notation for the central extension of a Kac-Moody algebra. $k$ is $c$-number valued in every irreducible representation of the Kac-Moody algebra.

Because

$$
\left[p_{n}^{i}, p_{m}^{j}\right]=1 \cdot n \cdot \delta_{n+m, 0} \delta^{i, j},
$$

we see that the currents (4.2) obey a $S \hat{O}(8)$-algebra with $k=1$. Since $\left\{p_{0}^{i}\right\} \equiv$ Cartan subalgebra in this representation of $S O(8) \subset S \hat{O}(8)$, we obtain

(a) $k=1$,

(b) $\{$ weight vectors of $S O(8)\}=\{\mathbf{p}$, with $|\mathbf{p}\rangle$ being the corresponding eigenstate $\}$,

(c) since all allowed types of momenta are of the $\left\{\lambda_{V}\right\}$ - or of the $\left\{\lambda_{s}\right\} /\left\{\lambda_{\bar{s}}\right\}$-type, we have the following classification of momentum eigenstates with respect to their $S O(8)$-transformation properties:

$$
\begin{aligned}
\left\{\left|\mathbf{p}=\lambda_{V}\right\rangle\right\}= & \text { the } 8 \text { weight-vector-eigenstates spanning } \\
& \text { the } 8 D \text { irreducible vector representation, } \\
\left\{\left|\mathbf{p}=\lambda_{s}\right\rangle\right\}= & \text { the } 8 \text { weight-vector-eigenstates spanning } \\
& \text { one of the two } 8 D \text { spinor representations, } \\
\left\{\left|\mathbf{p}=\lambda_{\vec{s}}\right\rangle\right\}= & \text { the } 8 \text { weight-vector-eigenstates spanning } \\
& \text { the other } 8 D \text { spinor representation. }
\end{aligned}
$$

In addition we note that

$$
\left\{\mathbf{p}= \pm e_{i} \pm e_{j} \mid i \neq j\right\} \cup\{\mathbf{0}\}=\text { all the roots of } S O(8) .
$$

Hence the highest root $\psi$ obeys $\psi^{2}=2$. 
(d) Combining (a) and (c), we see that the level $x$ of the Kac-Moody-algebrarepresentation furnished by (4.2) is given by

$$
x:=\frac{2 k}{\psi^{2}}=1 .
$$

Now, since $S O(8)$ is simply laced and because of (4.3), it is shown in [6] that

obey

$$
\begin{aligned}
& \mathscr{L}(z):=\frac{1}{2} \sum_{(i, j)=1}^{\operatorname{rank} S O(8)} \times T^{i j}(z) T^{i j}(z) \times \frac{\times}{\times} \equiv \times \times P(z)^{2 \times}, \\
& \overline{\mathscr{L}}(z):=\frac{\operatorname{rank} S O(8)}{\operatorname{dim} S O(8)} \cdot \frac{1}{2} \sum_{(i, j)=1}^{\operatorname{dim} S O(8)} \times T^{i j}(z) T^{i j}(z) \times
\end{aligned}
$$

$$
\mathscr{L}(z)=\overline{\mathscr{L}}(z),
$$

where $\times$ × $\times$ denotes "bosonic" normal ordering, i.e. with respect to $T_{m}^{i j}$.

Equation (4.5) says that the two $2 D$ quantum field theories described by the respective generators of their Virasoro algebras, $\mathscr{L}$ and $\overline{\mathscr{L}}$, defined on the same Hilbert space, are quantum equivalent in a highest weight (i.e. $L_{0}$ is bounded from below) unitary representation of the Virasoro algebras.

Moreover, we get from [6] that

where

$$
\overline{\mathscr{L}}(z) \cdot \frac{\operatorname{rank} S O(8)^{-1}}{\operatorname{dim} S O(8)^{-1}}=Q_{M} \cdot L(z),
$$

$$
L(z)=\frac{1}{2} \circ z \frac{d H^{j}}{d z} H^{j \circ}+\varepsilon \cdot 8,
$$

for all purely imaginary $8 D$ irreducible representations $i\left(T^{i j}\right)_{k l}$ of $S O(8) . Q_{M}$ is the value of the quadratic Casimir operator of the representation under consideration, $Q_{M}=\sum_{(i, j)=1}^{\operatorname{dim} S O(8)}\left(i T^{i j}\right)^{2}$. Thus $Q_{M}$ equals 7 , since the fermion operators $H^{i}(z)$ are in the vector representation of $S O(8)$. Finally

Thus

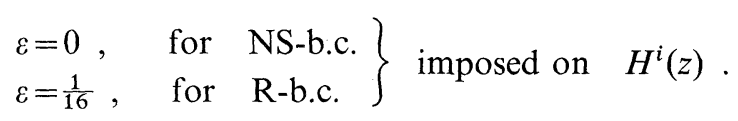

$$
\frac{1}{2} \times\left(P \cdot e_{j}\right)\left(P \cdot e_{j}\right) \times \frac{1}{2} \circ z \frac{d H^{j}}{d z}(z) H^{j}(z) \circ+\left\{\begin{array}{l}
\frac{1}{2}: \mathrm{R} \\
0: \mathrm{NS} .
\end{array}\right.
$$

Calculating the moments, $L_{m}$, of $\mathscr{L}(z)=\sum_{m \in Z} L_{m} z^{-m}$, we get

$$
\mathrm{R}: \quad L_{m}=\frac{1}{2} \sum_{n \in Z} \times{ }_{\times}^{\times} \alpha_{m+n}^{I} \alpha_{-n}^{I} \times \frac{1}{\times}=\frac{1}{2} \sum_{k \in Z} \circ H_{m+k}^{i} H_{-k}^{i} \circ(-k-m)+\frac{1}{2} \delta_{m, 0},
$$

NS: $\quad L_{m}=\frac{1}{2} \sum_{n \in Z} \times \alpha_{m+n}^{I} \alpha_{-n}^{I} \times \frac{1}{2} \sum_{r \in Z+\frac{1}{2}} \stackrel{\circ}{ } H_{m+r}^{i} H_{-r}^{i} \circ(-r-m)$. 
This shows the important equivalence of the free bosonic with the free fermionized theory. We note that the contribution of the 4 compactified dimensions to the normal ordering constants $c=\bar{c}=1$ in the bosonic string will be equal in both the boson and the fermion picture. Thus we may set

$$
L_{0}=\frac{1}{2} p^{2}+\sum_{n>0} \alpha_{-n}^{i} \alpha_{n}^{i}=\sum_{v} H_{-v}^{j} H_{v}^{j} \cdot v+\left\{\begin{array}{l}
\frac{1}{2}: \mathrm{R} \\
0: \mathrm{NS} .
\end{array}\right.
$$

The difference between the ground state energies, zero in the Neveu-Schwarz- and $\frac{1}{2}$ in the Ramond-sector, correctly accounts for the different vacua (3.7), (3.8).

Now we come to the last part of the construction of the NSR-models. First, we take the $d=10, D-d \geqq 5$, not necessarily consistent free bosonic string. We also assume that $\tau \in \mathbb{R}$. Secondly, any 4 of the $D-d$ compactified or (for open strings) remaining dimensions shall generate our $H_{v}^{i}$. Third, we choose arbitrarily a pure momentum eigenstate (i.e. eigenstate of $p_{L}, p_{R}$ or $\tilde{p}$ ), which we denote by $\left|\mathbf{v}_{h}\right\rangle$, subject to the sole condition $\mathbf{v}_{h}^{2}=1$, in the $D-d-4 \geqq 1$ dimensions. (We call $\left|\mathbf{v}_{h}\right\rangle$ the "hidden" vacuum for reasons to become clear.) Thus we select one of the many candidate Hilbert spaces $H=\mathscr{H}_{L} \otimes \mathscr{H}_{R} \otimes\left|p^{i}\right\rangle_{i=2, \ldots, d-1}$ or $H=\mathscr{H} \otimes\left|p^{i}\right\rangle_{i=2, \ldots, d-1}$, where

$$
\begin{aligned}
\mathscr{H}^{\#} & \left.=\left\{\left|\alpha^{\#}\right\rangle_{i=2, \ldots, 9} \otimes\left(\mathbf{H}_{-v}^{j \#}|v\rangle\right)_{I=1, \ldots, 4}\right) \otimes\left|\mathbf{v}_{h}^{\#}\right\rangle_{I \geqq 5}\right\}, \\
\mathscr{H} & \left.=\left\{|\alpha\rangle_{i=2, \ldots, 9} \otimes\left(\mathbf{H}_{-v}^{j}|v\rangle\right)_{I=1, \ldots, 4}\right) \otimes\left|\mathbf{v}_{h}\right\rangle_{I \geqq 5}\right\},
\end{aligned}
$$

and \# indicates left and right movers.

Now let us rewrite, for the purely bosonic compactified strings, Eqs. (2.7), (2.8), (2.10), and (2.12) on these Hilbert spaces $H$ :

closed:

$$
\begin{aligned}
& \frac{1}{8} m^{2}=N^{i}+N^{I}+\frac{1}{2}\left(p_{R}^{I}\right)^{2}-\frac{1}{2} \equiv N^{i}+L_{0}^{I}-\frac{1}{2}, \\
& \frac{1}{8} m^{2}=\bar{N}^{i}+\bar{N}^{I}+\frac{1}{2}\left(p_{L}^{I}\right)^{2}-\frac{1}{2} \equiv \bar{N}^{i}+\bar{L}_{0}^{I}-\frac{1}{2},
\end{aligned}
$$

open:

$$
\frac{1}{8} m^{2}=N^{i}+N^{I}+\frac{1}{2}(\tilde{p})^{2}-\frac{1}{2} \equiv N^{i}+L_{0}^{I}-\frac{1}{2},
$$

closed:

$$
\alpha_{m}^{-}=\frac{2}{p^{+}}\left(L_{m}^{i}+L_{m}^{I}\right), \quad \bar{\alpha}_{m}^{-}=\frac{2}{p^{+}}\left(\bar{L}_{m}^{i}+\bar{L}_{m}^{I}\right)
$$

open:

$$
\alpha_{m}^{-}=\frac{2}{p^{+}}\left(L_{m}^{i}+L_{m}^{I}\right)
$$

where $I$ indicates the 4 nontrivial compactified or remaining dimensions.

Plugging our fermionized expressions into these equations, identifying $H_{v}^{i}$ with $d_{n}^{i}$ or $b_{r}^{i}[1]$, since $\left\{H_{v}^{i}\right\}$ obey the required commutation rules and $\left(H_{v}^{i}\right)^{+}=H_{-v}^{i}$, we then get immediately the $d=D=10$ open and closed spinning strings, namely the NSR models:

$$
\text { closed } \quad \begin{aligned}
\quad \mathrm{NS} & : \frac{1}{8} m^{\# 2}=\sum_{\substack{n>0 \\
i=2, \ldots, 9}} \alpha_{-n}^{\# i} \alpha_{n}^{\# i}+\sum_{r>0} b_{-r}^{\# i} b_{r}^{\# i} \cdot r-\frac{1}{2}, \\
\mathrm{R}^{\#}: \frac{1}{8} m^{\# 2} & =\sum_{\substack{n>0 \\
i=2, \ldots, 9}} \alpha_{-n}^{\# i} \alpha_{n}^{\# i}+\sum_{n>0} d_{-n}^{\# i} d_{n}^{\# i} \cdot n,
\end{aligned}
$$


constraint: $m^{2}=\bar{m}^{2}$,

open:

$$
\begin{aligned}
\mathrm{NS}: & \frac{1}{8} m^{2}=\sum_{n>0} \alpha_{-n}^{i} \alpha_{n}^{i}+\sum_{r>0} b_{-r}^{i} b_{r}^{i} \cdot r-\frac{1}{2}, \\
\mathrm{R}: \frac{1}{8} m^{2} & =\sum_{n>0} \alpha_{-n}^{i} \alpha_{n}^{i}+\sum_{n>0} d_{-n}^{i} d_{n}^{i} \cdot n,
\end{aligned}
$$

closed:

$$
\begin{aligned}
\alpha_{m}^{\#-}= & \frac{1}{p^{+}} \sum_{n} \alpha_{m+n}^{\# i} \alpha_{-n}^{\# i}+\left(\frac{1}{p^{+}} \sum_{n} d_{m+n}^{\# j} d_{-n}^{\# j}(-m-n)\right. \\
& \text { or } \left.\frac{1}{p^{+}} \sum_{r} b_{m+r}^{\# j} b_{-r}^{\# j}(-m-r)\right),
\end{aligned}
$$

open:

$$
\alpha_{m}^{-}=\text {analogous to }(4.17) \text {, }
$$

where \# denotes left or right movers; the reader may convince him-(her-)self easily of the fact that the factors $(-m-k)$ or $(-m-r)$ in $(4.17),(4.18)$ are equivalent to $(-m / 2-k)$ or $(-m / 2-r)$ (used in [1]) or to the factors accurring in (2.7).

Of course we do not get directly the constraint-equation for $d_{v}^{\#-}$. It can be constructed using (2.7), where all operators needed are available now.

The groundstates are given by [cf. notation in (4.9)]:

$$
\begin{aligned}
\text { any R-sector: } & \left\{|\boldsymbol{0}\rangle \otimes\left(\left|\lambda_{s}\right\rangle \text { or }\left|\lambda_{\bar{s}}\right\rangle\right) \otimes\left|\mathbf{v}_{h}\right\rangle\right\}, \\
\text { any NS-sector: } & \left\{|\boldsymbol{0}\rangle \otimes|\mathbf{0}\rangle \otimes\left|\mathbf{v}_{h}\right\rangle\right\} .
\end{aligned}
$$

Observe that, since the fermionization procedure of Sect. 3 ultimately depends only on the scalar products of the orthonormal basis vectors $e_{i}$, and since the commutation relations of the $p^{\#}, q^{\#}, \alpha_{n}^{\#}$ do not single out some fixed directions either, we can choose any other orthonormal frame $\left\{\tilde{e}_{i}\right\}$ instead of $\left\{e_{i}\right\}$, e.g. $\left\{\lambda_{s}\right\}$ or $\left\{\lambda_{\bar{s}}\right\}$ thanks to triality. Thus, similar results hold if we start with $B\left(\lambda_{s}, z\right)$ or $B\left(\lambda_{\bar{s}}, z\right)$, and we easily get

$$
\begin{aligned}
& \left.\left\{B_{-1 / 2}^{\lambda_{s}}|\mathbf{0}\rangle\right\}=\left\{\mid \lambda_{s}, \alpha^{I}=0\right)\right\}, \\
& \left\{B_{-1 / 2}^{\lambda_{\bar{s}}}|\mathbf{0}\rangle\right\}=\left\{\left|\lambda_{\bar{s}}, \boldsymbol{\alpha}^{I}=0\right\rangle\right\},
\end{aligned}
$$

thus having explicitly created the R-groundstates out of the unique NS-groundstate. We note that the set $\left\{B_{-1 / 2}^{\lambda_{s}}, B_{-1 / 2}^{\lambda_{\bar{s}}}\right\}$ is an important ingredient in constructing the fermion emission vertex, in our framework.

Last, we write down the expressions for the Lorentz generators [1]. The interesting ones are the generators built from $b_{r}^{i}, d_{n}^{i}$ in the transverse directions:

and

$$
\begin{aligned}
& J^{i j}=L^{i j}\left(\alpha_{n}^{i}, q^{i}, p^{i}\right)+K_{0}^{i j}, \\
& K_{m}^{i j}=-\frac{i}{2} \sum_{n} \circ d_{m+n}^{k}\left(T^{i j}\right)_{k l} d_{-n}^{l} \circ, \quad m \in Z,
\end{aligned}
$$

or

$$
\circ d_{0}^{k} d_{0}^{l} \circ:=d_{0}^{k} d_{0}^{l}
$$

$$
K_{m}^{i j}=-\frac{i}{2} \sum_{r} \stackrel{\circ}{\circ} b_{m+r}^{k}\left(T^{i j}\right)_{k l} b_{-r}^{l \circ}, \quad m \in Z
$$

for right movers, or open strings. 
Comparing (4.21) with (4.2), we note that the physically relevant $S \hat{O}(8)$, generated by $K_{m}^{i j}$, and the $S \hat{O}(8)$ generated by the fermionic currents $T_{m}^{i j}$ are equivalent, i.e. $K_{m}^{i j} \equiv-T_{m}^{i j}, \forall m$, and the structure constants of the $K-S O(8)$ $=-$ those of $T-S O(8)$.

Thus the momentum operators $\left\{p \cdot e_{i}\right\}=\left\{p \cdot \tilde{\lambda}_{V}\right\}^{7}$ form a Cartan subalgebra also for the transverse Lorentz group, and we conclude immediately that the transformation properties of the groundstates under the transverse Lorentz group are as follows (cf. Eqs. (4.1a)-(4.3)):

(a) the NS-vacuum $|\mathbf{p}=\mathbf{0}\rangle$ is a singlet,

(b) the R-vacua are the weight-vector-eigenstates of both chiral irreducible spinor representations:

$$
p \cdot e_{i}\left|\lambda_{s}\right\rangle=\left(e_{i} \cdot \lambda_{s}\right)\left|\lambda_{s}\right\rangle, \quad \text { etc. }
$$

The same conclusion can be drawn if one uses Eqs. (3.7), (3.9), (3.20) to obtain that

(a) $\left\{H_{0}^{i}\right\}$ act on $\left\{\left|\lambda_{s}\right\rangle \oplus\left|\lambda_{\bar{s}}\right\rangle\right\}$ as the 16-dimensional representation of the Clifford-algebra.

(b) $\left\{H_{0}^{i}\right\}$ act on all massive states obtained from $|v\rangle$, i.e. $\left\{H_{-n_{1}}^{i_{1}} \ldots H_{-n_{m}}^{i_{m}}|v\rangle\right\}$, also purely as $\gamma$-matrices.

(c) $K_{0}^{i j}$ built from $H_{n}^{i}$ acts on $\left\{\left|\lambda_{s}\right\rangle \oplus\left|\lambda_{\bar{s}}\right\rangle\right\}$ as $K_{0 s}^{i j} \oplus K_{0 \bar{s}}^{i j}$, realizing both chiral sectors of the $S O(8)$ spinor representation.

In conclusion we found: with intermediate use of $2 D$ Euclidean space, i.e. allowing $\tau \in \mathbb{C}$, we have explicitly constructed all possible NSR-models from bosonic string theory on all admissible Hilbert spaces which are subspaces of the bosonic string. We have chown how to identify the various groundstates and how to realize them in terms of bosonic states and have verified their physical $S O(8)$ properties. Using appropriate GSO-projections in the NS- and R-sectors [1], we can readily identify various $10 \mathrm{D}$ supersymmetric subspaces of the bosonic string, since all states obtained by the action of $\left\{H_{-v}^{\# i}\right\}$ on $|v\rangle$ are linear combinations of $\left|\mathbf{p}^{\#}, \alpha^{\#}\right\rangle$-states.

Note that the full anticommuting structure characterising the NSR-models could not be implemented using only a commuting structure.

As an example we describe what we call the "minimal" embedding of the superstring in the bosonic string. "Minimal" refers to the fact that the embedding can be achieved using only $D=15$ bosonic dimensions, which is somewhat remarkable since naively one expects that $D$ should be equal to 19 at least, because we need 4 bosonic dimensions to construct $\left\{d_{n}^{i}\right\}$ and 4 to get $\left\{b_{r}^{j}\right\}$. But because $D\left(H_{n}^{i}\right) \perp D\left(H_{r}^{j}\right), D=15$ is sufficient. Thus we take $D=15, d=10$ and choose the common "hidden" vacuum $\left|v_{h}\right\rangle$ of the NS- and the R-sector in the $15^{\text {th }}$ dimension. Consequently the operators $p^{+}, p^{i}, q^{-}, q^{i}, \alpha_{n}^{\# i}, b_{r}^{\# i}$ and $d_{n}^{\# i}$ are realized using the following bosonic dimensions (see Table 1, p. 493).

Of course the subspaces we have chosen are stable under the action of all physically relevant operators.

Finally we want to comment on the interacting string. The expressions for the vertex operators and propagators can be found e.g. in [10]. We then see that

\footnotetext{
${ }^{7}$ We take $\left\{\tilde{\lambda}_{V}\right\}$ to be $\left\{e_{i}\right\}$ here, and afterwards, more generally, $\{\tilde{\lambda}\}=4$ linearly independent vectors of $\{ \pm \lambda\}$, and $\{\tilde{\lambda}\}$ shall be positively oriented
} 
Table 1

\begin{tabular}{lll}
\hline Bosonic dimensions & Spinning string dimensions & Operators \\
\hline$i=0,1$ & $i=0,1$ & $p^{+}, q^{-}$ \\
$i=2, \ldots, 9$ & $i=2, \ldots, 9$ & $\alpha_{n}^{\# i}, q^{i}, p^{i}$ \\
$I=1, \ldots, 4$ & $i=2, \ldots, 9$ & $b_{r}^{\# i}$ \\
$I=1, \ldots, 4$ & $j=2, \ldots, 9$ & $d_{n}^{\# j}$ \\
\hline
\end{tabular}

(a) All NSR-propagators can be identified with the bosonic one, since e.g.

$$
\begin{aligned}
\left(L_{0}^{\#}-1\right) & \equiv L_{0}^{\#}-\frac{1}{2}, & & \text { on any NS subspace } \\
& \equiv L_{0}^{\#}, & & \text { on any R subspace },
\end{aligned}
$$

(b) with our conventions the vertex for tachyon emission for bosonic strings is

$$
V^{\#}\left(p, z^{\#}\right)=z^{\#-p^{2} / 8}: e^{i p / 2 \cdot Q \#}:
$$

with conformal weight $\Delta=1 \Leftrightarrow p^{2} \equiv m^{2}=-8$.

Taking special values for $p$, e.g. $p=\left(k^{+}, k^{-}, k^{i}, 2 e_{i}, 0\right)$, we get $p^{2}=k^{2}-4$ and thus $k^{2}=-4$. So we have

$$
\begin{aligned}
V^{\#}\left(\left(k, 2 e_{i}\right), z^{\#}\right) & =z^{\#-\frac{k^{2}}{2}}: e^{i \frac{k}{2} \cdot Q^{\#}}: z^{\# \frac{e_{i}^{2}}{2}}: e^{i e_{i}^{I} \cdot Q^{\# I}}: \\
& =z^{\#-\frac{k^{2}}{s}}: e^{i \frac{k}{2} \cdot Q^{\#}}: B^{\#}\left(e_{i}, z^{\#}\right),
\end{aligned}
$$

where $Q^{I}$ is given by (3.1), (3.2). Taking linear combinations of $V^{\prime} s$, we then get the tachyon $\left(k^{2}=-4\right)$ emission vertex of the NS-sector in light-cone-gauge and in a Lorentz frame where $k^{+}=0$ :

$$
V_{\mathrm{NS}}(k, z)=\mathrm{const} \cdot\left(k^{i} \cdot H^{i}(z)\right) \cdot z^{-\frac{k^{2}}{8}} \cdot: e^{i k / 2 \cdot Q}: \text {. }
$$

Similar results are expected to hold for all boson-emission vertices of the NSRmodels.

(c) This simple procedure does not work in the R-sector, because we would have to modify the vertex operators of bosonic string theory in order to get the fermionic vertices of the NSR-models.

Thus it does not seem possible to simplify the calculation of correlation functions involving external fermions by just reading them off from scattering amplitudes of bosonic string theory. Furthermore, there are also some drawbacks affecting the correlations of external NSR-bosonic states: First, for reasons of selfconsistency, we must include projection operators in the bosonic string correlation functions which guarantee that only the states belonging to the chosen NSR-subspace propagate. Secondly, as demonstrated in the case of the tachyon vertex, each NSR-bosonic vertex operator which we can construct from the bosonic string vertex operators will in general be obtained in an individual Lorentz frame. Thus even after inserting the suitable projection operators into the bosonic string correlation functions we can get the corresponding correlators of external NSR-bosons at most for a very limited number of them. 


\section{Possible Superstrings Formulated on the Ramond Sector}

As promised in the Introduction, in this section we focus our attention on the superstring candidates of [5], i.e. on the Ramond sector of the $d=10, D \geqq 15$ partially fermionized bosonic strings, and we choose $\mathbf{v}_{h}^{2}=1$. The reason is as follows: The integer fermionic modes of the Ramond-sector provide us with a mass formula which, in the case of $d=10, D \geqq 15$ and $\mathbf{v}_{h}^{2}=1$, is the expression expected to hold for every superstring which is formulated in the new formalism and exhibits only the physical degrees of freedom. Correct chiralities of the states and of the operators are two necessary requirements which have to be satisfied by a string theory which "wants" to become, for instance in the present case, a superstring. Thus we will investigate the chiral properties of the states and of the $d_{n}^{i}$-operators if one uses not only the standard Lorentz generators $K_{m}^{i j}$, but also their triality-transformed versions (see below). Because it seems to be rather difficult to construct the $10 D$ supercharges, we will not be able to prove that our candidates really are superstring theories.

With this in mind we reexamine the Ramond-sector constructed in the previous sections, i.e. where the anticommuting coordinates were obtained from $\left\{B\left(\lambda_{V}, z\right)\right\}$, defined on $D_{R}(B)$, with mass-formula

$$
\frac{1}{8} m^{\# 2}=\sum_{n>0} \alpha_{-n}^{\# i} \alpha_{n}^{\# i}+\sum_{n \geqq 0} d_{-n}^{\# i} d_{n}^{\# i} \cdot n,
$$

and where the transverse Lorentz generators are given by Eq. (4.21). Because the Cartan subalgebra of the physically relevant $S O(8)$ is generated by

we know that the groundstates

$$
\left\{\tilde{\lambda}_{V} \cdot p\right\}
$$

$$
\left\{\left|\lambda_{s}\right\rangle\right\}, \quad\left\{\left|\lambda_{\bar{s}}\right\rangle\right\}
$$

form the two irreducible spinor representations of $S O(8)$. Thus we cannot build on them a supersymmetric spectrum. (This is the reason why we have to introduce the NS-fields; namely in order to get the vector-like groundstates.)

We now wish to generalize this construction. One motivation is the fact that the fermionic operators in a superstring theory based on the new formalism behave like spinors under the action of the Lorentz group. Because the $d_{n}^{i}$-part of the transverse Lorentz group is based on the $S \hat{O}(8)$ generated by $\left\{-T_{m}^{i j}\right\}$ [Eq. (4.2)], and because the $\left\{-T_{m}^{i j}\right\}$ themselves are induced by $\left\{\left(T^{i j}\right)_{k l}\right\}$ which specify the transformation properties of the $\left\{d_{n}^{i}\right\}$ under the transverse Lorentz group completely, we are interested in exchanging the vector-like $\left(T^{i j}\right)_{k l}$ with spinor-like $\left(T^{i j}\right)_{k l}$.

Thus let us consider the following $S O(8)$ triality-rotations,

and

$$
v \mapsto s: i\left(T^{i j}\right)_{k l} l_{(4.1 \mathrm{~b})} \mapsto \frac{i}{4}\left[\gamma^{i}, \gamma^{j}\right]_{k l} \equiv i\left(\sigma^{i j}\right)_{k l}
$$

$$
v \mapsto \bar{s}:\left.i\left(T^{i j}\right)_{k l}\right|_{(4.1 \mathrm{~b})} \mapsto \frac{i}{4} \overline{\left[\gamma^{i}, \gamma^{j}\right]_{k l}} \equiv i\left(\bar{\sigma}^{i j}\right)_{k l},
$$

where the $8 D$ irreducible representations $i\left(\sigma^{i j}\right)_{k l}$ and $i\left(\sigma^{i j}\right)_{k l}$ are of type $s,(5.2)$, and of type $\bar{s},(5.3)$, which means that their weight-vectors are $\left\{\lambda_{s}\right\}$ and $\left\{\lambda_{\bar{s}}\right\}$ respectively. 
It is convenient to write

$$
\begin{aligned}
& \left(T^{i j}\right)_{k l}(v):=\left(T^{i j}\right)_{k l} \text { of Eq. (4.1b), } \\
& \left(T^{i j}\right)_{k l}\left(s^{\#}\right):=\left(\sigma^{\# i j}\right)_{k l} \quad \text { of type } s^{\#},
\end{aligned}
$$

where $\sigma^{\#}$ and $s^{\#}$ stand for $\sigma$ or $\bar{\sigma}$ and $s$ or $\bar{s}$ respectively.

The transformations (5.2) and (5.3) are given by real automorphisms [9], i.e.

$$
\left(T^{i j}\right)_{k l}\left(s^{\#}\right)=A^{\# i j}{ }_{m n}\left(T^{m n}\right)_{k l}(v) \text {, }
$$

where $A{ }^{\# i j} \in \mathbb{R}$. Moreover the Cartan generators $\left\{i T^{1}(v), \ldots, i T^{4}(v)\right\}$ are mapped as follows [9]:

$$
\begin{aligned}
& i T^{1}\left(s^{\#}\right)=\frac{i}{2}\left(T^{1}+T^{2}+T^{3} \mp T^{4}\right)(v), \\
& i T^{2}\left(s^{\#}\right)=\frac{i}{2}\left(T^{1}+T^{2}-T^{3} \pm T^{4}\right)(v), \\
& i T^{3}\left(s^{\#}\right)=\frac{i}{2}\left(T^{1}-T^{2}+T^{3} \pm T^{4}\right)(v), \\
& i T^{4}\left(s^{\#}\right)=\frac{i}{2}\left(\mp T^{1} \pm T^{2} \pm T^{3}+T^{4}\right)(v),
\end{aligned}
$$

where the upper sign refers to $s$, the lower one to $\bar{s}$.

After these group theoretic remarks, we return to Sect. 4. Under the trialityrotations (5.2), (5.3) the currents (4.2) obviously are transformed as

$$
T^{i j}(z, v) \mapsto T^{i j}\left(z, s^{\#}\right),
$$

and by (5.6) the Cartan-generators are mapped as

$$
\left\{\lambda_{v} \cdot P\right\} \mapsto\left\{\lambda_{s^{*}} \cdot P\right\}
$$

Now, because the moments of $\left\{\lambda_{\mathrm{s}} \cdot \mathrm{P}\right\}$ also obey the abelian Kac-Moody-algebra with $k=1$, we know that Eqs. (4.3)-(4.5) still hold.

Secondly, because the map (5.5) is a real automorphism connecting the 3 inequivalent $8 D$ irreducible representations of $S O(8)$, we know by [6] that also Eq. (4.6) is still valid. Of course $Q_{M}$ equals 7 in all these 3 cases.

Third, because

$$
\underset{\times}{\times}\left(\tilde{\lambda}_{v} \cdot P\right)\left(\tilde{\lambda}_{v} \cdot P\right) \underset{\times}{\times}=\underset{\times}{\times}\left(\tilde{\lambda}_{s} \cdot P\right)\left(\tilde{\lambda}_{s} \cdot P\right) \underset{\times}{\times}=\underset{\times}{\times}\left(\tilde{\lambda}_{\bar{s}} \cdot P\right)\left(\tilde{\lambda}_{\bar{s}} \cdot P\right) \underset{\times}{\times},
$$

we conclude that in the $S O(8)$-rotated theory Eqs. (4.7) and (4.8) are also correct. Thus the constructions of Sect. 4, (4.1)-(4.8), can be carried out independent of the $S O(8)$ conjugation class, i. e. independently of whether we make use of $T^{i j}(z, v)$, of $T^{i j}(z, s)$ or of $T^{i j}(z, \vec{s})$. The states $\left\{\left|\lambda_{s}\right\rangle\right\},\left\{\left|\lambda_{s}\right\rangle\right\}$ are thus the groundstates in all these 3 cases.

Furthermore, the Lorentz generators (4.21) become

$$
K_{m}^{i j}\left(s^{\#}\right)=-T_{m}^{i j}\left(s^{\#}\right)=-\frac{i}{8} \sum_{n} \circ d_{m-n}^{k}\left[\gamma^{i}, \gamma^{j}\right]_{k l}^{\#} d_{n}^{l \circ},
$$

for right movers, or open strings. 
Of course the Lorentz algebra still closes, as in the case of $K_{m}^{i j}(v)$, since all 3 types of $K_{m}^{i j}$ obey the same $S \hat{O}(8)$-algebra, and because of (5.7).

Thus we have found: The Ramond-type operators $d_{n}^{i}$ transform as $8_{s \#}$ under $K_{0}^{i j}\left(s^{\#}\right)$, and the chiralities of the groundstates are given by

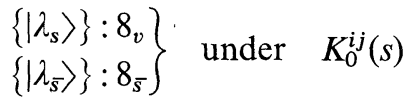

$$
\begin{aligned}
& \left.\begin{array}{l}
\left\{\left|\lambda_{s}\right\rangle\right\}: 8_{s} \\
\left\{\left|\lambda_{\vec{s}}\right\rangle\right\}: 8_{v}
\end{array}\right\} \quad \text { under } K_{0}^{i j}(\vec{s})
\end{aligned}
$$

which can be easily verified using Eq. (5.6b).

This looks very promising because now we can have spinor- and vector-like groundstates and fermionic operators of the third chirality-type. So we get the supersymmetric spectrum required for candidate superstrings.

Let us finally consider a second kind of generalization of the Ramond-model (5.1). We could have fermionized the 4 bosonic directions using any orthonormal frame $\left\{\tilde{e}_{j}\right\}=\left\{R e_{i} \mid R \in O(4)\right\}$. Special cases are $\left\{\tilde{e}_{j}\right\}=\left\{\tilde{\lambda}_{s}\right\}$ and $\left\{\tilde{e}_{j}\right\}=\left\{\tilde{\lambda}_{\bar{s}}\right\}$. It is easy to verify that all the results of Sect. 3, 4 and 5 hold, if we replace, according to these basis transformations,

$$
\begin{aligned}
& D\left(B\left(\lambda_{V}, z\right)\right)_{R}= \begin{array}{l}
\left\{\left|\lambda_{s}+\Lambda_{R}\right\rangle\right. \\
\left.\oplus\left|\lambda_{\bar{s}}+\Lambda_{R}\right\rangle\right\}
\end{array} \mapsto D\left(B\left(\lambda_{s}, z\right)\right)_{R}=\begin{array}{l}
\left\{\left|\lambda_{V}+\Lambda_{R}\right\rangle\right. \\
\left.\oplus\left|\lambda_{\bar{s}}+\Lambda_{R}\right\rangle\right\}
\end{array} \\
& \text { or } \mapsto D\left(B\left(\lambda_{\bar{s}}, z\right)\right)_{R}=\begin{array}{l}
\left\{\left|\lambda_{s}+\Lambda_{R}\right\rangle\right. \\
\left.\oplus\left|\lambda_{V}+\Lambda_{R}\right\rangle\right\}
\end{array}
\end{aligned}
$$

$$
\text { and } \quad \circ B\left(\lambda_{V}, z\right) B\left(-\lambda_{V}, z\right) \circ=\lambda_{V} \cdot P(z) \quad \text { (Eq. (3.13)) }
$$

becomes $: B\left(\lambda_{s^{*}}, z\right) B\left(-\lambda_{s^{*}}, z\right) \circ=\lambda_{s^{*}} \cdot P(z)$.

We denote the fermionic fields $H(z)$, the inducing representations $(T)$, the currents $T(z)$ and their moments $T_{m}$ by $H^{j}(z), H^{a}(z), H^{\bar{a}}(z) ;\left(T^{i j}\right)_{k l},\left(T^{a b}\right)_{c d},\left(T^{\bar{a} \bar{b}}\right)_{\bar{c} \bar{d}}$; etc., in the case of $B\left(\lambda_{V}, z\right), B\left(\lambda_{s}, z\right)$ and $B\left(\lambda_{\bar{s}}, z\right)$ respectively.

Summarizing (5.9) and these remarks we obtain the following Table 2:

Table 2

\begin{tabular}{ll}
\hline $\begin{array}{l}S \hat{O}(8) \text {-representation (4.2) } \\
\text { (or, equivalently) }\end{array}$ & Transformation property of the \\
Lorentz-algebra & Ramond groundstates \\
induced by & $\left(\left\{\left|\lambda_{v}^{s}\right\rangle\right\},\left\{\left|\lambda_{\bar{s}}^{\bar{s}}\right\rangle\right\}\right)$ under \\
& $K^{i j}$ \\
& $K_{0 a b}^{i j}$ \\
& $\bar{a} \bar{b}$ \\
\hline$(T)(v)\left(\right.$ i.e. $\left.\left(T^{i j}\right)_{k l}(v),\left(T^{a b}\right)_{c d}(v),\left(T^{\bar{a} \bar{b}}\right)_{\bar{c} \bar{d}}(v)\right)$ & $\left(8_{s}, 8_{\bar{s}}\right)$ \\
$(T)(s)$ & $\left(8_{v}, 8_{\bar{s}}\right)$ \\
$(T)(\bar{s})$ & $\left(8_{s}, 8_{v}\right)$ \\
\hline
\end{tabular}

Here, the shorthand in the upper right half of the Table $2(5.10)$ means that $K^{i j}$ is acting on $\left\{\left|\lambda_{s}\right\rangle\right\},\left\{\left|\lambda_{\bar{s}}\right\rangle\right\}$, and that $K^{a b}$ and $K^{\bar{a} \bar{b}}$ act on $\left\{\left|\lambda_{v}\right\rangle\right\},\left\{\left|\lambda_{\bar{s}}\right\rangle\right\}$ and $\left\{\left|\lambda_{s}\right\rangle\right\},\left\{\left|\lambda_{v}\right\rangle\right\}$ respectively. So we learn that one always needs $(T)(s)$ or $(T)(\vec{s})$ in order to get candidate superstrings. 
Using (5.10) we are in a position to compare the bosonic states making up the GSO-projected NSR-model and our candidate superstring. We assume that the massless states of both theories are given by

$$
\left\{\left|\lambda_{v}\right\rangle\right\},\left\{\left|\lambda_{s}\right\rangle\right\}
$$

Because (5.11) uniquely fixes one of the three possible domains of definition of the operators $H_{n}$, we are led to investigate the action of the superstring operators $H_{n}^{\bar{a}}$ on the states (5.11). For the candidate superstring we choose $(T)(\vec{s})$, and for the NSRstring we choose $(T)(v)$ as the inducing representation of the Lorentz algebra. It then follows that the states (5.11) transform as $\left(8_{v}, 8_{s}\right)$ in both theories.

In order to perform the correct GSO-projection (onto $G=-1$ states), we must act with an odd power of $\left(b_{-r}^{j}\right)$-operators on $|0\rangle$, and with even and odd powers of $\left(d_{-n}^{i}\right)$-operators on $\left\{\left|\lambda_{s}\right\rangle\right\}$ and $\left\{\left|\lambda_{\bar{s}}\right\rangle\right\}$ respectively. Taking into account (3.17), it is easy to check that at the first two mass levels we get the following set of basis vectors (apart from cocycle factors):

groundstates: NSR-superstring

Superstring candidate

$$
\left\{\left\{b_{-1 / 2}^{i}|0\rangle\right\} \cong\left\{\left|\lambda_{v}\right\rangle\right\}\right\} \cup\left\{\left|\lambda_{s}\right\rangle\right\}
$$

$1^{\text {st }}$ mass level: NSR-superstring

$$
\left\{\left|\lambda_{v}\right\rangle\right\} \cup\left\{\left|\lambda_{s}\right\rangle\right\}
$$

$$
\begin{aligned}
\left\{\left\{b_{-1 / 2}^{i} b_{-1 / 2}^{j} b_{-1 / 2}^{k}|0\rangle\right\}\right. & \cup\left\{b_{-3 / 2}^{j}|0\rangle\right\} \triangleq\left\{\left|\lambda_{v}\right\rangle \otimes \alpha_{-1}^{I}|0\rangle\right\} \\
& \left.\cup\left\{\left| \pm e_{k} \pm e_{m} \pm e_{n}\right\rangle ; k \neq m \neq n \neq k\right\}\right\} \\
& \cup\left\{\left\{\alpha_{-1}^{i} b_{-1 / 2}^{j}|0\rangle\right\} \cong\left\{\left|\lambda_{v}\right\rangle \otimes \alpha_{-1}^{i}|0\rangle\right\}\right\} \\
& \cup\left\{\left\{d_{-1}^{i}\left|\lambda_{\bar{s}}\right\rangle\right\} \triangleq\left\{\left|\lambda_{v}+\lambda_{\bar{s}}\right\rangle\right\}\right\} \\
& \cup\left\{\left\{\alpha_{-1}^{i}\left|\lambda_{s}\right\rangle\right\}=\left\{\left|\lambda_{s}\right\rangle \otimes \alpha_{-1}^{i}|0\rangle\right\}\right\}
\end{aligned}
$$

Superstring candidate

$$
\begin{aligned}
\left\{\left\{H_{-1}^{\bar{a}}\left|\lambda_{v}\right\rangle\right\} \triangleq\right. & \left.\left\{\left|\lambda_{v}+\lambda_{\bar{s}}\right\rangle\right\}\right\} \\
& \cup\left\{\left\{H_{-1}^{\bar{a}}\left|\lambda_{s}\right\rangle\right\} \hat{\equiv}\left\{\left|\lambda_{v}\right\rangle \otimes \alpha_{-1}^{I}|0\rangle\right\}\right. \\
& \left.\cup\left\{\left| \pm e_{k} \pm e_{m} \pm e_{n}\right\rangle ; k \neq m \neq n \neq k\right\}\right\} \\
& \cup\left\{\left|\lambda_{v}\right\rangle \otimes \alpha_{-1}^{i}|0\rangle\right\} \\
& \cup\left\{\left|\lambda_{s}\right\rangle \otimes \alpha_{-1}^{i}|0\rangle\right\} .
\end{aligned}
$$

Thus we get the same states with the same chiralities. It is very plausible that this result holds true at each mass level ${ }^{8}$. We will comment on the implications of our calculation during the following

Discussion. On the Hilbert spaces $\mathscr{H}^{i}, \mathscr{H}^{a}, \mathscr{H}^{\bar{a}}$, e.g.

$$
\mathscr{H}^{i}=\left|p^{i}\right\rangle_{i=2, \ldots 9} \otimes\left\{|\boldsymbol{\alpha}\rangle^{\#} \otimes\left(\mathbf{H}_{-n}^{j^{*}}\left|\lambda_{s}\right\rangle \cup \mathbf{H}_{-m}^{j^{\#}}\left|\lambda_{\bar{s}}\right\rangle\right) \otimes\left|\mathbf{v}_{h}^{\#}\right\rangle\right\},
$$

\footnotetext{
${ }^{8}$ Note added: Using the formulation of [11] this statement can in fact be proved without too much effort
} 
we have constructed, by an $S O(8)$-triality transformation, in light-cone gauge, an automatically chiral string theory with supersymmetric spectrum. Here, the fermionic operators $\mathscr{H}_{n}^{i, a, \bar{a}}$ transform vectors into spinors and are thus expected to be part of the supersymmetry generators. For a given type of $\mathscr{H}$, i. e. for $\mathscr{H}^{i}$ or $\mathscr{H}^{a}$ or $\mathscr{H}^{\bar{a}}$, the set of the groundstates is fixed. But their chiralities are not fixed and can be adjusted choosing the appropriate $8 D$ irreducible representation of $S O(8)$ which induces the $S \hat{O}(8)$ current-algebra. There is the conventional Lorentz algebra which closes also after triality transformation. All the massive states are obtained by the action of $\left\{\mathscr{H}_{n}^{i, a, \bar{a}}\right\}$ and $\left\{\alpha_{-m}^{i}\right\}$ on the groundstates.

Having established the equivalence (with respect to the spectrum of physical states) between the $8 D$ superstring candidates (in Green-Schwarz formalism) and the NSR-superstring, it only remains to construct the $10 D$ supercharges $Q^{\bar{a}}$. Together with the Lorentz generators $K^{\bar{a}}(\bar{s})$ they should provide the full $10 D$ super Lorentz algebra in the Green-Schwarz formalism. Although finding the $Q^{\bar{a}}$ should not be an impossible task [in view of (4.20) and of the SUSY properties of $H_{n}^{\bar{a}}$ ], it seems to be a rather laborious one. Having in mind the results of [1], it is obvious that we also need $\gamma^{+}$and $\gamma^{-}$, for which there is simply no room left in our $8 D$ world. We see that our $8 D$ language has its limitations.

This is manifestly so if we want to compare our would-be superstring, which explicitly exhibits all the physical degrees of freedom, with the superstring in its "new" formulation, because the "new" formalism never solves explicitly for the physical components; one always uses the full 32 -spinor and the $10 D$ Clifford algebra in its $32 \mathrm{D}$ reducible representation, taking into account various projection properties.

Summarizing we restate the remaining open problem: In order to prove the $10 D$ supersymmetry of our candidate superstring theories we need the supercharges $Q^{a}$, which are unknown so far.

\section{Outlook}

It is not obvious whether it will be possible to incorporate the fermionization in string field theory and consequently the embeddings of the NSR-strings and of the heterotic strings in a bosonic string. The reason is that fermionization and the creation of the gauge algebra are highly nonlinear transformations on component oscillators, and that in both cases one needs to invoke additional structure (operator cocycles) in order to get the desired subtheories. So we do not expect that purely bosonic string fields are capable of producing superstring and heterotic fields without additional ingredients.

Functional integrals look much more promising, because the partition function of bosonic strings restricted to the $10 \mathrm{D}$ supersymmetric subspaces must yield the superstring expression. But, of course, the question mark concerning the embedding of the interacting superstring is still present.

\section{Appendix A}

The commutation relation (3.9c) can be easily derived using Eq. (5.3) of [3]. However, for the sake of completeness, we will sketch its verification. 
Taking into account (3.1), (3.3), and (3.4), Eq. (3.9a) is a well-known result, and consequently we get

$$
\begin{aligned}
& {\left[\frac{1}{2} p^{2}, B(e, z)\right]=z^{\frac{e^{2}}{2}} e^{i e \cdot Q<}\left[\frac{1}{2} p^{2}, e^{2 i e \cdot q}\right] z^{e p} e^{i e Q>}} \\
& =z^{\frac{e^{2}}{2}} e^{i e \cdot Q<} e^{2 i e \cdot q}\left(e \cdot p+\frac{1}{2} e^{2}\right) z^{e p} e^{i e Q>} \\
& =B(e, z) \cdot\left(e \cdot p+\frac{1}{2} e^{2}\right) \text {. }
\end{aligned}
$$

Because $L_{0}=\frac{1}{2} p^{2}+N$, we now calculate $[N, B(e, z)]$. Remember that the BakerCampbell-Hausdorff formula for operators $A$ and $B$ with $[A, B] \in \mathbb{C}$ is given by

Writing

$$
e^{A} e^{B}=e^{A+B+1 / 2[A, B]} .
$$

$$
\alpha_{n}^{I}=\left.\frac{d}{d y} e^{\alpha_{n}^{I} y}\right|_{y=0}
$$

and because $\left[\alpha_{m}^{I}, Q_{<}^{J}\right]=0$, for $m<0$, we see that

$$
\begin{aligned}
{\left[N, e^{i e \cdot Q<}\right] } & =\sum_{n>0} \alpha_{-n}^{I}\left[\alpha_{n}^{I}, e^{i e \cdot Q<}\right] \\
& =\left.\sum_{n>0} \alpha_{-n}^{I} \frac{d}{d y}\left\{e^{\alpha_{n}^{I} y} e^{i e \cdot Q<}-e^{i e Q}<e^{\alpha_{n}^{I} y}\right\}\right|_{y=0} \\
& =\left.\sum_{n>0} \alpha_{-n}^{I} \frac{d}{d y}\left\{e^{\alpha_{n}^{I} y-\sum_{k<0} \frac{e \alpha_{k}}{k} z^{-k}+\frac{1}{2} y e^{I} z^{n}}-e^{\alpha_{n}^{I} y-\sum_{k<0} \frac{e \alpha_{k}}{k} z^{-k}-\frac{1}{2} y e^{I} z^{n}}\right\}\right|_{y=0}
\end{aligned}
$$

where in the last step we used (A.2) and the commutation relations (2.13), and thus ${ }^{9}$

$$
\begin{aligned}
{\left[N, e^{i e Q<}\right]=} & \sum_{n>0} \alpha_{-n}^{I}\left\{\int_{0}^{1} d s e^{s i e \cdot Q_{<}}\left(\alpha_{n}^{I}+\frac{1}{2} e^{I} z^{n}\right) e^{(1-s) i e \cdot Q_{<}}\right. \\
& \left.-\int_{0}^{1} d s e^{s i e \cdot Q_{<}}\left(\alpha_{n}^{I}-\frac{1}{2} e^{I} z^{n}\right) e^{(1-s) i e \cdot Q_{<}}\right\} \\
= & \sum_{n>0} \alpha_{-n}^{I} e^{I} z^{n} e^{-\sum_{k<0} \frac{e^{J} \alpha_{k}^{J}}{k} z^{-k}} \\
= & z \frac{d}{d z}\left(e^{-\sum_{k<0} \frac{e \alpha_{k}}{k} z^{-k}}\right) \equiv z \frac{d}{d z}\left(e^{i e \cdot Q_{<}}\right)
\end{aligned}
$$

By the same method we obtain

$$
\left[N, e^{i e Q>}\right]=z \frac{d}{d z} e^{i e Q>}
$$

${ }^{9}$ We use the fact that

$$
\frac{d}{d y} e^{A(y)}=\int_{0}^{1} d s e^{s \cdot A(y)}\left(\frac{d}{d y} A(y)\right) e^{(1-s) A(y)}
$$


Combining (A.1), (A.4), and (A.5) we get

$$
\left[L_{0}, B(e, z)\right]=z \frac{d}{d z} B(e, z)
$$

Now we plug in the Laurent-expansion (3.4) for $B(e, z)$. The result is

$$
\left[L_{0}, B_{v}^{e}\right]=-v B_{v}^{e} .
$$

By Eq. (A.1) we know that

$$
\left[\frac{1}{2} p^{2}, B_{v}^{e}\right]=B_{v}^{e}\left(e \cdot p+\frac{1}{2} e^{2}\right),
$$

and since $L_{0}=\frac{1}{2} p^{2}+N$, we get

$$
\left[N, B_{v}^{e}\right]=B_{v}^{e}\left(-v-\frac{1}{2}-e \cdot p\right),
$$

because $e^{2}=1$. This proves all the commutation relations (3.9a)-(3.9c).

Acknowledgements. It is a pleasure to thank J. Fröhlich and D. Wyler for carefully reading the manuscript and for various stimulating discussions.

\section{References}

1. For a review see e.g. Schwarz, J.H.: Superstring theory. Phys. Rep. 89, 223 (1982), and references therein

2. Coleman, S.: Quantum sine-Gordon equation as the massive Thirring model. Phys. Rev. D 11, 2088 (1975);

Witten, E.: Non-abelian bosonization in two dimensions. Commun. Math. Phys. 92, 455 (1984)

3. Goddard, P., Olive, D.: DAMTP 83/22 (1983)

4. Freund, P.G.O.: Superstrings from 26 dimensions? Phys. Lett. B 151, 387 (1985)

5. Casher, A., Englert, F., Nicolai, H., Taormina, A.: Consistent superstrings as solutions of the $D=26$ bosonic string theory. Phys. Lett. B 162, 121 (1985)

6. Olive, D.: Imperial TP $84-85 / 33$

7. Gross, D.J., Harvey, J.A., Martinec, E., Rohm, R.: Heterotic string theory (I). The free heterotic string. Nucl. Phys. B 256, 253 (1985)

8. Brink, L., Di Vecchia, P., Howe, P.: A locally supersymmetric and reparametrization invariant action for the spinning string. Phys. Lett. B 65, 471 (1976)

9. Georgi, H.: Lie Algebras in particle physics; Frontiers in physics, Vol. 54. Benjamin Cummings 1982

10. Scherk, J.: An introduction to the theory of dual models and strings. Rev. Mod. Phys. 47, 123 (1975)

11. Englert, F., Nicolai, H., Schellekens, A.: CERN-TH 4360/86

Communicated by K. Gawedzki

Received February 4, 1987; in revised form March 10, 1987 